\title{
Genome-wide meta-analysis of genetic susceptible genes for Type 2 Diabetes
}

\author{
Paul J Hale ${ }^{1,2}$, Alfredo M López-Yunez ${ }^{3}$, Jake Y Chen ${ }^{1,2,4,5^{*}}$ \\ From The International Conference on Intelligent Biology and Medicine (ICIBM) \\ Nashville, TN, USA. 22-24 April 2012
}

\begin{abstract}
Background: Many genetic studies, including single gene studies and Genome-wide association studies (GWAS), aim to identify risk alleles for genetic diseases such as Type II Diabetes (T2D). However, in T2D studies, there is a significant amount of the hereditary risk that cannot be simply explained by individual risk genes. There is a need for developing systems biology approaches to integrate comprehensive genetic information and provide new insight on T2D biology.
\end{abstract}

Methods: We performed comprehensive integrative analysis of Single Nucleotide Polymorphisms (SNP's) individually curated from T2D GWAS results and mapped them to T2D candidate risk genes. Using protein-protein interaction data, we constructed a T2D-specific molecular interaction network consisting of T2D genetic risk genes and their interacting gene partners. We then studied the relationship between these T2D genes and curated gene sets.

Results: We determined that T2D candidate risk genes are concentrated in certain parts of the genome, specifically in chromosome 20. Using the T2D genetic network, we identified highly-interconnected network "hub" genes. By incorporating T2D GWAS results, T2D pathways, and T2D genes' functional category information, we further ranked T2D risk genes, T2D-related pathways, and T2D-related functional categories. We found that highlyinterconnected T2D disease network "hub" genes most highly associated to T2D genetic risks to be PI3KR1, ESR1, and ENPP1. The well-characterized TCF7L2, contractor to our expectation, was not among the highest-ranked T2D gene list. Many interacted pathways play a role in T2D genetic risks, which includes insulin signalling pathway, type II diabetes pathway, maturity onset diabetes of the young, adipocytokine signalling pathway, and pathways in cancer. We also observed significant crosstalk among T2D gene subnetworks which include insulin secretion, regulation of insulin secretion, response to peptide hormone stimulus, response to insulin stimulus, peptide secretion, glucose homeostasis, and hormone transport. Overview maps involving T2D genes, gene sets, pathways, and their interactions are all reported.

Conclusions: Large-scale systems biology meta-analyses of GWAS results can improve interpretations of genetic variations and genetic risk factors. T2D genetic risks can be attributable to the summative genetic effects of many genes involved in a broad range of signalling pathways and functional networks. The framework developed for T2D studies may serve as a guide for studying other complex diseases.

\footnotetext{
* Correspondence: jakechen@iupui.edu

${ }^{1}$ School of Informatics, Indiana University-Purdue University, Indianapolis, IN, USA

Full list of author information is available at the end of the article
} 


\section{Background}

Type 2 Diabetes (T2D) is a complex metabolic disease that affects 25.8 million Americans in 2011, according to statistics reported by Centers for Disease Control and Prevention (CDC). T2D occurs when the body develops resistance to insulin due to the malfunction of insulin producing $\beta$-cells. The developmental process of T2D involves a complex interplay between genetic and environmental factors. However, it is not clear how the underlying genetic defects give rise to T2D pathogenesis over time. Recent T2D genetic study results, particularly those from genome-wide association studies (GWAS), have yielded insights to the molecular mechanisms and underlying genetic risk factors of T2D [1]. Among the many risk genes identified are: transcription factor 7-like 2 (TCF7L2)[2-4], peroxisome proliferator-activated receptor gamma (PPARG) [5-7], and potassium inwardlyrectifying channel, subfamily J, member 11 (KCNJ11) $[5,6]$.

These GWAS results were challenging to interpret. Many single nucleotide polymorphisms (SNPs) identified from GWAS tend to show strong sample biases and may not extrapolate from one population to another. In T2D, only approximately $28 \%$ of the disease heritability may be explained by identified individual SNPs that showed statistical significance in these samples/population-a problem known as missing heritability [8]. The combined effects of multiple risk SNP's can increase the overall odds ratio of $\mathrm{T} 2 \mathrm{D}$ by 1.24 per allele for up to 8.68 among 18 risk alleles in one study [9] and by 1.265 per allele in another study [6]. The additive effect suggests the presence of molecular system structures that are essential to T2D pathogenesis.

To confirm the presence of molecular systems structures that may better explain missing heritability problems for T2D, we adopted a Systems Biology approach to studying T2D genetic risk gene networks as a whole rather than the risk genes individually. Prior to this study, several reports $[10,11]$ examined genes implicated T2D differential expressions in affected tissues. In this study, we used T2Dassociated SNP information curated from the Type 2 Diabetes Genetic Association Database (T2DGADB), which integrated comprehensively reported SNPs, their odds ratios, population description, and all related metadata from various T2D GWAS performed worldwide [12]. We further annotated individual SNPs collected from T2DGADB with information from the DbSNP database [13], including information such as nearby genes, Chromosomal location, gene functional class, and base changes. To create a model for T2D genetic risk gene molecular systems structure, we built a gene interaction network seeded by T2D risk genes collected from T2DGADB and expanded with high-confidence protein interaction data collected from the Human Annotated and Predicted Protein Interaction database (HAPPI) [14]. We also ranked risk genes in the network according to these high confidence interactions.

\section{Methods}

T2D risk SNPs and risk genes data collection and curation Data from both the $\mathrm{ftp}$ site and web pages of T2DGADB were downloaded. On the ftp site, only a gene list and a SNP list without annotation were available for download. Therefore, the complete information from individual web pages of T2DGADB was extracted into a single Excel file manually. Data entries with dbSNP SNP cross-references were kept and entries without dbSNP SNP cross-reference information were removed from this study. Gene annotation information is derived from the VEGA [15] database. The Excel file was imported into the ORACLE $11 \mathrm{~g}$ database for subsequent efficient database querying.

\section{Statistical significance testing of T2D risk SNPs and risk genes}

Once we collected information integrated from T2DGADB and dbSNP, we applied standard hyper-geometric tests (using an R software package called phyper) to the data set to determine which chromosomes were over-represented/ under-represented. We determined significance on three data sets. First, the distribution of dbSNP of all human SNP's in current build of known origins were compared against that of risk SNP's across all chromosomes. Second, the distribution of genes where the risk SNPs can be mapped to were compared against that of all the genes across all chromosomes. Third, the distribution of proteincoding genes where the risk SNPs can be mapped to were compared against that of all the protein-coding genes across all chromosomes.

\section{Construction and analysis of the T2D risk genes network}

To generate risk gene network, we incorporated protein interaction data from the HAPPI database. The database integrated protein-protein interactions comprehensively from STRING [16], OPHID [17], BIND [18], HPRD [19], and MINT [20] to generate an overall confidence score for each interaction. High-quality interactions (e. g., confidence score $>0.8$ in the database version of the HAPPI) are strongly correlated with physical binding based protein interaction relationships. Using risk genes from T2DGADB as input, we queried the HAPPI database and retrieved related high-quality protein interactions involving risk genes as one of the interaction partner to build a risk gene protein interaction network. The retrieved genes in the T2D risk gene subnetwork were then ranked with the following network gene ranking method, which was originally introduced in [21]: 


$$
r_{p}=k * \ln \left(\sum_{q \in N E T} \operatorname{conf}(p, q)\right)-\ln \left(\sum_{q \in N E T} N(p, q)\right)(1)
$$

In this equation, $r_{p}$ score measures the relative significance of a gene/protein in the subnetwork. $p$ and $q$ are proteins in the subnetwork. $k$ is a constant set at two for our purposes. conf $(p, q)$ is the confidence score of the interaction between the two proteins provided by the HAPPI database and is 0 if $p$ and $q$ do not interact. $\mathrm{N}(\mathrm{p}, \mathrm{q})$ is 1 if the proteins have an interaction and is 0 otherwise. With all of this we are able to generate a network map of all involved genes.

Using $r_{p}$ score as a base score that considers T2D disease context in the molecular interaction network, we further defined a modified score, $r_{\text {mod }}$, to take into account of genes with strong genetic ties to T2D.

$$
r_{\text {mod }}=r_{p} \times \sqrt{P_{\text {count }}} \times O R_{a v j}
$$

$r_{\text {mod }}$ adjusts $r_{p}$ score of any risk gene with both the count of populations, $P_{\text {count }}$, in which significant risk SNPs were identified, and the average odds ratio $\left(O R_{a v j}\right)$ of reported risk SNPs found in these studies. Genes containing only one significant study can still be adjusted using the formula provided here. In constructing the final T2D risk gene network, we modified the original network to exclude studies in which the risk genes were determined to be insignificant $\left(r_{p}\right.$ score $\left.<2\right)$ before we calculated $r_{\text {mod }}$ scores for risk genes. Cytoscape software was used to visualize network relationships among genes and gene sets (to be described next).

\section{Construction and analysis of the T2D risk genes network}

To further sift the results and explore functional connections, we also mapped genes onto known gene sets. For this purpose, we used DAVID [22,23] to search for enriched KEGG [24] pathways. We also used GARNET [25] to identify enriched Gene Ontology categories and their relationships.

\section{Results}

\section{SNPs identified from various T2D GWAS}

Based on T2DGADB, we collected 4358 T2D SNP entries that cover 518 PubMed articles reporting T2D Genomewide association studies (GWAS) results worldwide. Since not all study reported statistics on all SNPs, there are only 3720 SNP entries with P-values, 2715 SNP entries with complete odds ratios, 2406 SNP entries with sample size and minor allele frequency values. All together, there are 1269 SNP entries with the abovementioned complete set of statistics. After comparing collected information against dbSNP entries manually, we validated 333 SNP gene annotations, re-annotated 11 SNP gene annotations, and flagged 140 additional genes that do not appear to be consistent with dbSNP curated locus information. The "mis-annotations" in T2DGADB were partly due to the presence of two genes, e.g., $\mathrm{ABCC} 8$ and $\mathrm{KCNJ} 11$, for some reported SNPs, therefore confounding manual curations. In other cases, gene symbols that are similar to each other, e.g., AGER and RAGE, which do not even appear on the same chromosome, also seemed to have been mixed up. The flagging of putative genes, e.g., LOC387761, according to dbSNP was performed, because we wanted to prioritize genes with known gene functions. In the end, we collected 4085 distinct SNP entries that cover 1539 SNPs in 370 different genes. Among these SNP entries, 598 SNPs from 255 different genes passed a P-Value significance cutoff of 0.05 .

\section{Observation of chromosomal specificity for T2D risk genes}

We calculated the distribution of curated T2D SNPs and genes across different chromosomes and showed the statistic significance using P-values in Table 1 . The results showed that chromosomes 1, 6, 11, 12, 17, 20 are overrepresented for T2D SNPs mapped to the chromosome, with chromosome 20 being most significant $(\mathrm{P}$-value $=$ 3.3E-38). To adjust for potential over-sampling of certain well-annotated T2D genes for genotyping studies in the

Table 1 P-value of Chromosomal Specificity Significant Test.

\begin{tabular}{rrrr}
\hline Chromosome & $\begin{array}{r}\text { P-value } \\
\text { (SNP) }\end{array}$ & $\begin{array}{r}\text { P-value (all } \\
\text { genes) }\end{array}$ & $\begin{array}{r}\text { P-value (coding } \\
\text { genes) }\end{array}$ \\
\hline 1 & $5.3 \mathrm{E}-06$ & 0.014 & 0.073 \\
2 & 0.99 & 0.61 & 0.56 \\
3 & 0.071 & $4.3 \mathrm{E}-03$ & 0.011 \\
4 & 0.99 & 0.21 & 0.12 \\
5 & 1.00 & 0.88 & 0.81 \\
6 & 0.039 & 0.47 & 0.58 \\
7 & 0.087 & 0.037 & 0.023 \\
8 & 1.00 & 0.70 & 0.60 \\
9 & 1.00 & 0.96 & 0.95 \\
10 & 0.15 & 0.38 & 0.38 \\
11 & $2.8 \mathrm{E}-04$ & 0.83 & 0.92 \\
12 & 0.022 & 0.55 & 0.60 \\
13 & 1.00 & 0.57 & 0.39 \\
14 & 1.00 & 1.00 & 1.00 \\
15 & 1.00 & 0.70 & 0.72 \\
16 & 0.65 & 0.25 & 0.84 \\
17 & 0.026 & 0.15 & 0.56 \\
18 & 1.00 & 0.15 & 0.47 \\
19 & 0.34 & 0.017 & 0.13 \\
20 & $7.0 \mathrm{E}-38$ & $6.1 \mathrm{E}-08$ & $2.4 \mathrm{E}-06$ \\
21 & 0.94 & 0.63 & 0.58 \\
22 & 0.98 & 1.00 & 1.00 \\
\hline & & &
\end{tabular}


GWAS results, we also report the significance testing results based on the distribution of genes that contained risk SNPs (namely "all genes") and the distribution of protein-coding genes that contained risk SNPs (namely "coding genes"). The results showed that chromosomes $1,3,7,19$, and 20 are over-represented for SNP-mapped T2D genes, with chromosome 20 being most significant $(\mathrm{P}$-value $=6.1 \mathrm{E}-08)$. Moreover, chromosomes 3, 7, and 20 are over-represented for SNP-mapped T2D proteincoding genes, with chromosome 20 still being most significant $(\mathrm{P}$-value $=2.4 \mathrm{E}-06)$. The results suggest that chromosome 20 contains an unusually large number of T2D risk SNPs across diverse GWAS, and the risk SNPs can be mapped to a unusually large number of risk genes, particularly risk protein-coding genes, which cannot be simply attributed to inherent genotyping bias among GWAS across different human populations. The result suggests that chromosome 20 may be a risk chromosome.

Contributing to this result are genes including Hepatocyte Nuclear Factor 4 Alpha (HNF4A) and Protein Tyrosine Phosphotase non-receptor type 1 (PTPN1), both of which have been extensively studied for their roles to T2D genetics. Linkage studies dating back to 1997 showed a modest association with this region of chromosome 20 [26,27], when whole genome data was not available. Particularly interesting is that the interplay between genetics and environment also may act on this chromosomal region, e.g., the epigenetic effect of diet on the promoter regions of HNF4A [28].

\section{Strong network centrality in T2D high-risk genes}

In Table 2, we show the original top-ranking T2D risk genes ordered by their $r_{p}$ scores, which we calculated based on T2D network connectivity information. After applying the filter " $r_{p}$ score $>=2$ " and re-constructing

Table 2 Top-ranking T2D risk genes ordered by their $\boldsymbol{r}_{\boldsymbol{p}}$ scores in the T2D risk gene protein interaction network.

\begin{tabular}{rrr}
\hline Rank & Gene & $\boldsymbol{r}_{\boldsymbol{p}}$ Score \\
\hline 1 & PI3KR1 & 114.09 \\
2 & ESR1 & 77.66 \\
3 & ENPP1 & 74.28 \\
4 & IL6 & 66.95 \\
5 & IL10 & 60.19 \\
6 & PRKAA2 & 56.47 \\
7 & PDE4B & 56.33 \\
8 & ADCY3 & 53.45 \\
9 & IRS1 & 48.65 \\
10 & CD14 & 46.16 \\
11 & GCK & 46.16 \\
12 & TCEB1 & 44.23 \\
13 & INSR & 41.39 \\
\hline
\end{tabular}

the T2D risk gene network, we derived a $r_{\text {mod }}$ score for each gene and show the network in Figure 1. The figure confirms that genes with high $r_{\text {mod }}$ scores are all network hubs with the resulting scores shown in Table 3. Genes with known $r_{\text {mod }}$ scores are shown to their node sizes and categorized by color. In Table 4, we listed topranking T2D genes ordered by $r_{\text {mod }}$ scores. Most of the top- 5 ranking genes still kept their relatively high ranks before $r_{\text {mod }}$ score calculations.

Our study suggests PI3KR1 may be the gene with highest T2D genetic risk associated. PIK3R1 is a regulatory subunit of the phosphoinositide-3-kinase, which is a protein known to be involved in insulin actions, cancer signaling, and cytokine signaling. While the coverage of the gene's functional relationship to T2D risks in T2DGADB is very limited (with one article only) [29], there is increasing evidence, including a recent SNP UTR study [30] and a mixed methods meta-analysis [31], that supports our finding.

The second highest-ranking gene in the T2D risk gene network by $r_{p}$ scores is ESR1, the estrogen receptor 1 gene. The gene encodes a transcription factor that responds to estrogen action and cancer, and will also form a heterodimer with ESR2. There are two articles cited in the T2DGADB database [32,33].

The third highest-ranking gene in the network is ENPP1, Ectonucleotide Pyrophosphatase/Phosphodiesterase 1, a trans-membrane glycoprotein involved in metabolism and has been shown to have an effect of insulin signaling and glucose metabolism [34]. ENPP1 was well studied among 20 GWAS-related publications collected through T2DGADB and 10 of those studies returned positive results in the population examined.

To demonstrate that the network hub genes are indeed functionally associated with T2D risks, we performed a t-test on the distribution of risk SNP per gene between the top ranked $25 \%$ of risk genes and the bottom $75 \%$ of risk genes. When ranks are given by the original $r_{p}$ scores, the results showed significant difference $(\mathrm{P}=$ 0.01 ) between the two groups for the reported risk SNP per gene raition $(3.06 \mathrm{SNP} /$ gene for the top $25 \%$ "hub genes" vs. $1.67 \mathrm{SNP} /$ gene for the bottom $75 \%$ "non-hub genes"). When ranks are given by the modified $r_{p}$ scores, the results showed even higher significant difference $(\mathrm{P}=$ $3.55 \mathrm{E}-4$ ) between the same two groups (3.74 SNP/gene for the top $25 \%$ "hub genes" vs. $1.45 \mathrm{SNP} /$ gene for the bottom $75 \%$ "non-hub genes").

\section{Functional heterogeneity and cohesion of T2D high-risk genes}

To gain an overview of functional categories represented by the T2D high-risk genes, we mapped these genes onto curated pathways. In Table 4, we list top significantly over-represented pathways identified by 


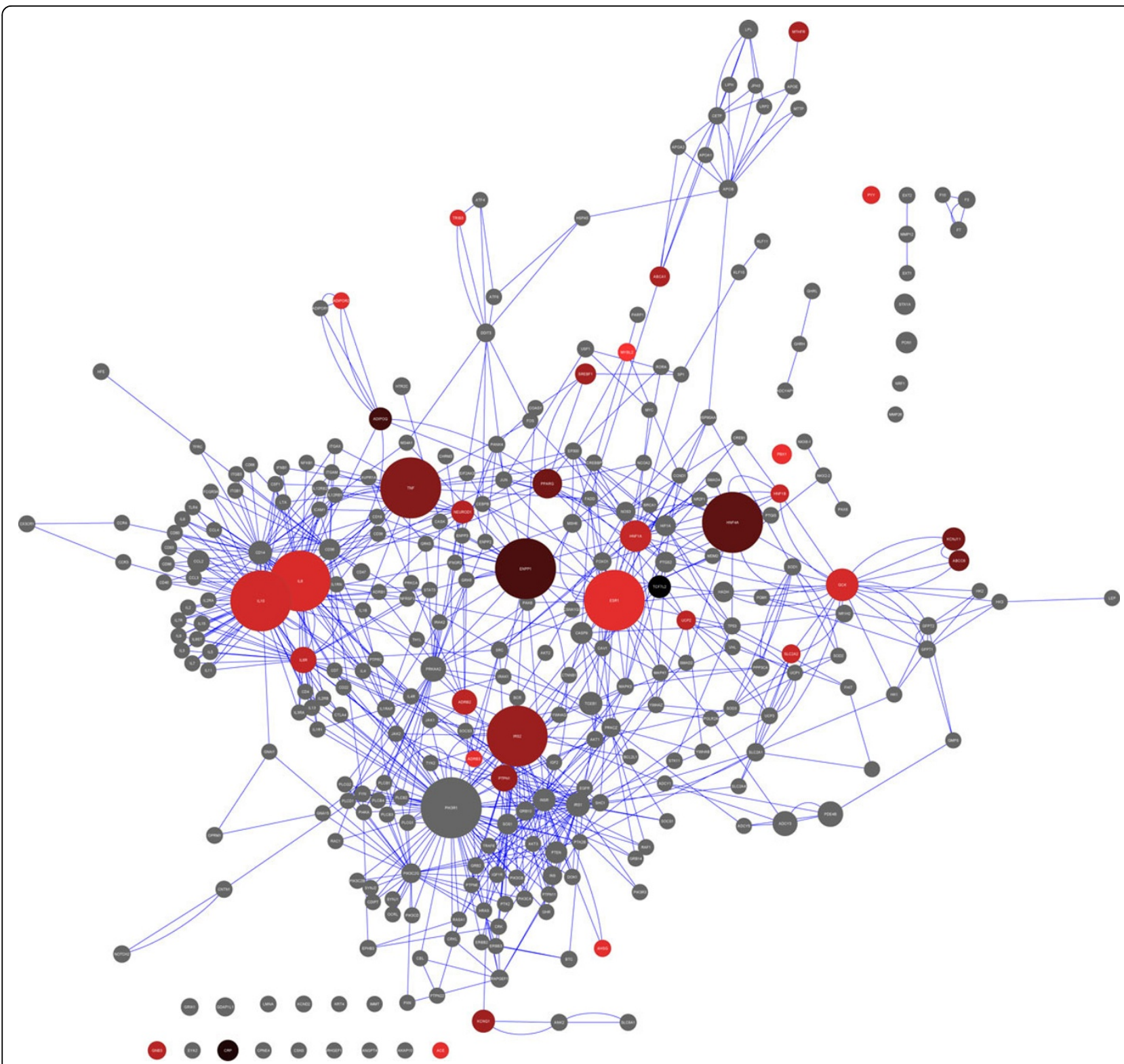

Figure 1 T2D risk gene network. Genes are represented as nodes drawn to the scale of their $r_{\text {mod }}$ scores. Higher-risk genes (high average odds ratio) are colored black and lower-risk genes (low average odds ratio) are colored red. Genes without significantly reported risk information are colored grey.

the DAVID software. Two highly ranked "novel" pathways identified (excluding known T2D pathways) are the Adipocytokine signaling and the PPAR signaling pathway.

From this result, we constructed T2D risk gene pathway interaction network (in Figure 2), if and only if interacting pathways involve 3 or more risk genes. The pathway interaction network showed distinct clusters involving cancer signaling. A few instead of many of identified risk genes may have contributed to the formation of these clusters. In the cancer pathway cluster,
CASP9 and PIK3R1 are both involved in 7 different cancer pathways, while SOS1 and TCF7L2 are both involved in 5 different pathways.

Using the GARNET software, we also identified highly enriched gene ontology (GO) categories (as shown in Table 5) and "crosstalk" between GO functional categories (as shown in Figure 3). All these results confirmed the high relevance of T2D risk genes to glucose-related metabolism and insulin-related hormonal regulations. Pathway analysis even revealed possible activation of pathways related to cancer/cell cycle controls. 
Table 3 Top-ranking T2D risk genes ordered by their $r_{\text {mod }}$ scores in the T2D risk gene protein interaction network.

\begin{tabular}{|c|c|c|c|c|}
\hline Rank by $r_{\text {mod }}$ Score & Gene & $r_{\text {mod }}$ Score & $r_{p}$ Score & Original Rank by $r_{p}$ Score \\
\hline 1 & ENPP1 & 430 & 74.3 & 3 \\
\hline 2 & ESR1 & 142 & 77.7 & 2 \\
\hline 3 & IL6 & 137 & 67.0 & 4 \\
\hline 4 & IL10 & 132 & 60.2 & 5 \\
\hline 5 & GCK & 119 & 46.2 & 11 \\
\hline 6 & HNF4A & 118 & 4.72 & $\gg 13$ \\
\hline 7 & PIK3R1 & 114 & - & 1 \\
\hline 8 & TNF & 110 & 3.75 & $\gg 13$ \\
\hline 9 & IRS2 & 110 & - & $>13$ \\
\hline 10 & HNF1A & 105 & - & $>13$ \\
\hline 11 & PPARG & 82.9 & 4.27 & $\gg 13$ \\
\hline 12 & PTPN1 & 69.8 & 3.69 & $\gg 13$ \\
\hline 13 & IL6R & 61.0 & - & $>13$ \\
\hline
\end{tabular}

\section{Discussion}

In this study, we showed our findings of T2D genetic risk gens. Genes from the chromosome 20 collectively accounted for the highest T2D genetic risks of all the chromosomes. However, the individual contribution of these chromosome 20 risk genes is relatively small. HNF4A as the most significant gene on chromosome 20 has a relatively small $r_{p}$ score of 4.72 , which is far lower than all $r_{p}$ scores shown for the top-ranking T2D risk genes in Table 2. Nonetheless, when all other information derived from GWAS results are integrated into the $r_{\text {mod }}$ score of 118, the significant contribution of HNF4A to $\mathrm{T} 2 \mathrm{D}$ risks becomes clear. The "missing inheritability" problem of $\mathrm{T} 2 \mathrm{D}$ genetic risks are therefore partially explained with the calculated integration of T2D molecular interaction network information and T2D genotypephenotype association study results.

In this study, the findings depend on the quality of underlying data that we integrated from network biology, GWAS results, and SNP annotations. The data is complex,

Table 4 Enriched pathways identified in the T2D risk genes.

\begin{tabular}{rrrr}
\hline Term & Count & $\%$ & P-value \\
\hline Adipocytokine signalling pathway & 15 & 7.01 & $6.21 \mathrm{E}-11$ \\
Type II diabetes mellitus & 12 & 5.61 & $2.18 \mathrm{E}-09$ \\
Insulin signalling pathway & 17 & 7.94 & $1.60 \mathrm{E}-08$ \\
Maturity onset diabetes of the young & 8 & 3.74 & $5.63 \mathrm{E}-07$ \\
PPAR signalling pathway & 9 & 4.21 & $9.11 \mathrm{E}-05$ \\
Calcium signalling pathway & 10 & 4.67 & $1.20 \mathrm{E}-02$ \\
Renal cell carcinoma & 6 & 2.80 & $1.60 \mathrm{E}-02$ \\
Aldosterone-regulated sodium reabsorption & 4 & 1.87 & $5.55 \mathrm{E}-02$ \\
VEGF signalling pathway & 5 & 2.34 & $7.43 \mathrm{E}-02$ \\
Adherens junction & 5 & 2.34 & $8.02 \mathrm{E}-02$ \\
Pathways in cancer & 12 & 5.61 & $8.68 \mathrm{E}-02$ \\
\hline
\end{tabular}

often derived from many different sources and groups. For example, TCF7L2, the most commonly studied gene in the collected data set, was covered by 45 GWAS, with up to a 3.4 odds ratio reported in a Finnish study [35]. However, such results face tremendous challenges in getting duplicated in other populations such as Japanese [36], Chinese [37], and Pima Indian [38]. In Table 6, we demonstrate the heterogeneity of results reported for the gene reported in different populations. Apparently, the gene is least significant in the "African American" population (average odds ratio is 1.19, among the lowest shown; yet it seems highly significant among Japanese and French, with average odds ratios being 1.57 and 1.62 respectively.

TCF7L2 was not among the highest ranked T2D genes, primarily due to the emphasis on the quality of protein interaction data that we bring in. The HAPPI database reported 238 protein interactions for TCF7L2 but only 11 of those were above the confidence threshold of 0.8 . This is in contrast to ENPP1, in which we identified 743 protein interactions from the HAPPI database and 87 of them passed our confidence threshold of 0.8 . The relatively low network centrality explained why TCF7L2 is not ranked at the top overall, although it is a population target for many T2D GWAS.

Future analysis that is built upon this work could benefit by integrating additional genomics and functional genomics information, e.g., available miRNA or mRNA expression information, available copy number variations results, and whole genome sequencing data.

\section{Conclusions}

Large-scale systems biology meta-analyses of GWAS results can improve interpretations of genetic variations and genetic risk factors. In this work, we determined that T2D candidate risk genes are located in higher concentration in certain parts of the genome, specifically in chromosome 20. Using the T2D genetic network, we identified 


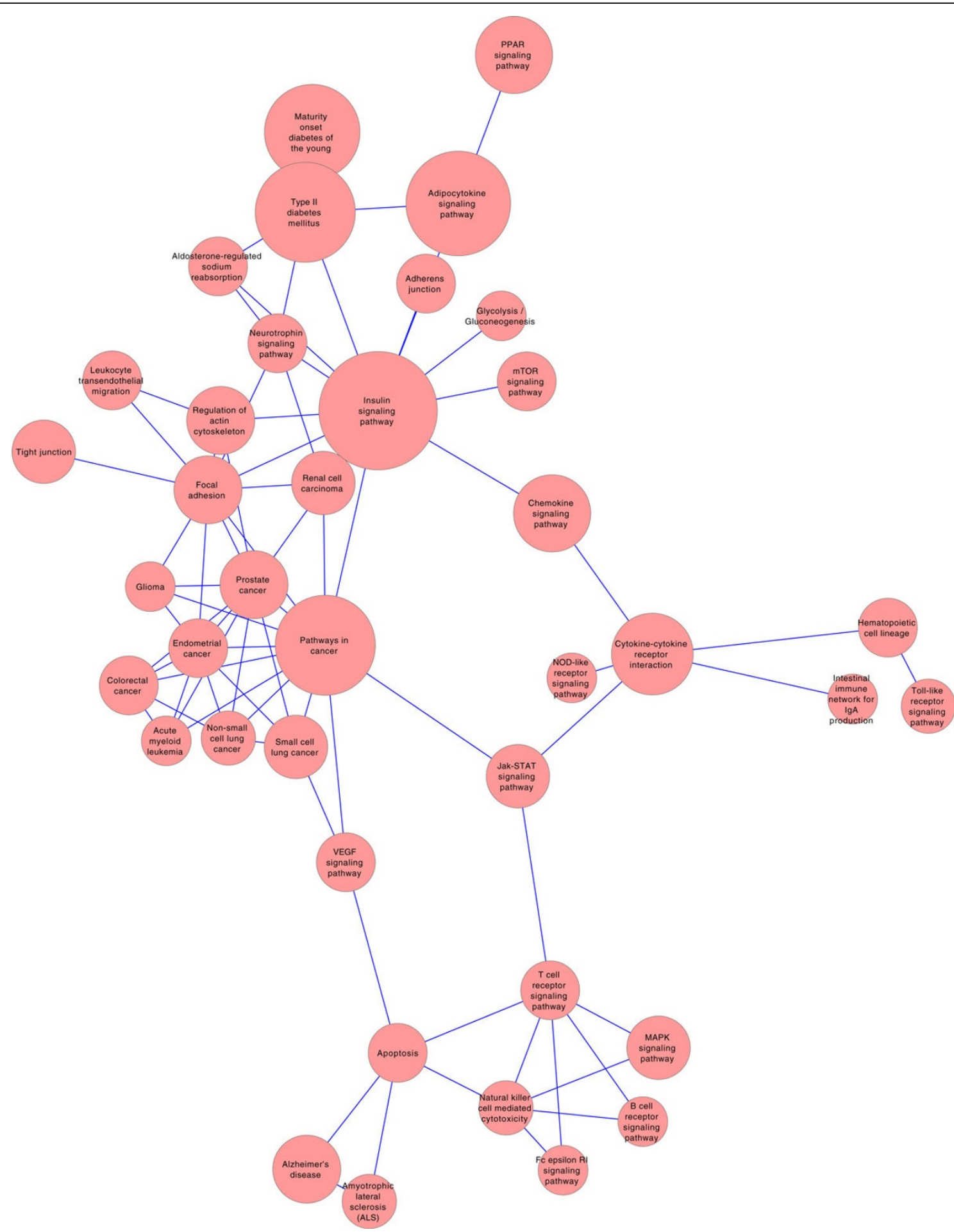

Figure 2 T2D risk gene pathway interaction network. Here, an edge will be created between two pathways, if and only if the pathways involved three of more risk genes.

highly interconnected network "hub" genes. By incorporating T2D GWAS results, T2D pathways, and T2D genes' functional category information, we further ranked T2D risk genes, T2D-related pathways, and T2D-related functional categories. Overview maps involving T2D genes, gene sets, pathways, and their interactions are all reported. Moreover, we demonstrate a computational framework built upon disease-specific data integration, 
Table 5 Enriched gene ontology categories identified in the T2D risk genes.

\begin{tabular}{|c|c|c|}
\hline Gene Ontology Term & Genes Involved & $P$ Value \\
\hline glucose homeostasis & 16 & $6.16 \mathrm{E}-09$ \\
\hline regulation of glucose transport & 12 & $6.61 \mathrm{E}-08$ \\
\hline regulation of insulin secretion & 15 & $6.59 \mathrm{E}-08$ \\
\hline glucose transport & 15 & 3.33E-07 \\
\hline peptide secretion & 19 & 2.82E-07 \\
\hline insulin secretion & 17 & $3.78 \mathrm{E}-07$ \\
\hline response to insulin stimulus & 22 & $2.42 \mathrm{E}-06$ \\
\hline response to peptide hormone stimulus & 27 & 3.02E-06 \\
\hline cellular response to hormone stimulus & 25 & 3.79E-06 \\
\hline hormone transport & 20 & $6.62 \mathrm{E}-06$ \\
\hline cholesterol transport & 12 & 8.92E-06 \\
\hline positive regulation of glucose import & 8 & $9.49 \mathrm{E}-06$ \\
\hline positive regulation of fatty acid metabolic process & 8 & $1.42 \mathrm{E}-06$ \\
\hline cellular response to insulin stimulus & 16 & 1.83E-05 \\
\hline negative regulation of macrophage derived foam cell differentiation & 6 & 2.02E-05 \\
\hline positive regulation of glucose metabolic process & 7 & $3.00 \mathrm{E}-05$ \\
\hline regulation of lipid metabolic process & 19 & $3.42 \mathrm{E}-05$ \\
\hline
\end{tabular}

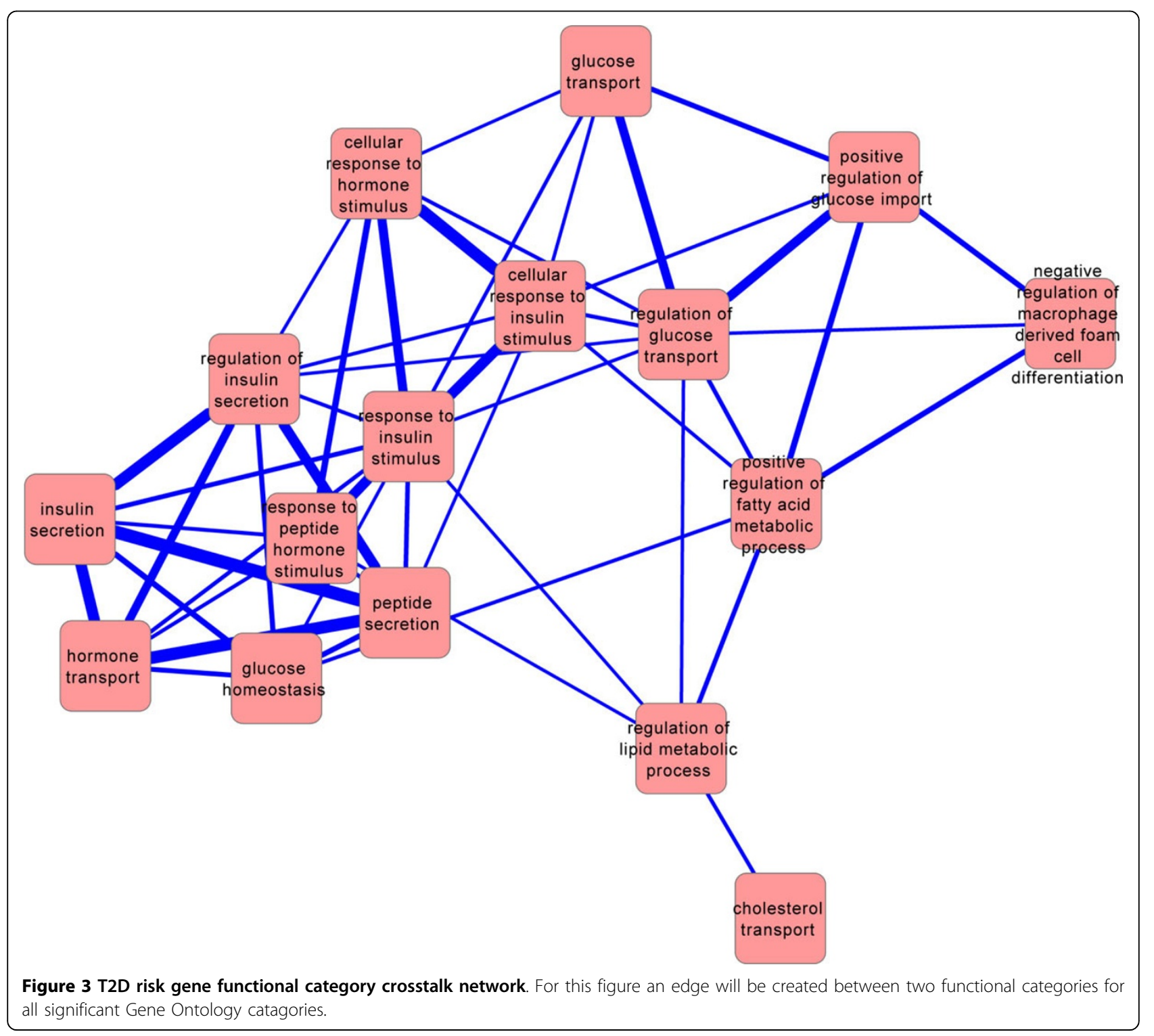


Table 6 GWAS results show population-specific effectiveness in using TCF7L2 for T2D genetic risk profiling.

\begin{tabular}{rrrrr}
\hline Population & Number of Studies & Average Odds Ratio & Maximum Odds Ratio & Minimum Odds Ratio \\
\hline American & 7 & 1.36 & 2.14 & 0.82 \\
Swedish & 6 & 1.49 & 2.15 & 1.08 \\
Finnish & 5 & 1.45 & 3.40 & 1.01 \\
UK & 5 & 1.50 & 2.47 & 2.08 \\
Japanese & 3 & 1.57 & 1.84 & 1.16 \\
French & 2 & 1.62 & 1.39 & 1.45 \\
African American & 2 & 1.19 & 1.51 & 1.24 \\
German & 2 & 1.37 & 1.96 & 1.29 \\
Dutch & 2 & 1.47 & 1.28 & 1.93 \\
Indian & 2 & 1.48 & 1.15 &
\end{tabular}

model construction, and data analysis. The framework developed for T2D studies may serve as a guide for studying other complex diseases.

\section{Acknowledgements}

This article has been published as part of BMC Systems Biology Volume 6 Supplement 3, 2012: Proceedings of The International Conference on Intelligent Biology and Medicine (ICIBM) - Systems Biology. The full contents of the supplement are available online at http://www.biomedcentral.com/ bmcsystbiol/supplements/6/S3.

\section{Author details}

${ }^{1}$ School of Informatics, Indiana University-Purdue University, Indianapolis, IN, USA. ${ }^{2}$ Indiana Center for Systems Biology and Personalized Medicine, Indianapolis, IN, USA. ${ }^{3}$ Alevio Medical Center, Indianapolis, IN, USA. ${ }^{4}$ Department of Computer \& Information Science, Purdue University, Indianapolis, IN, USA. ${ }^{5}$ Wenzhou Medical College, Wenzhou, Zhejiang Province, China.

\section{Authors' contributions}

JYC conceived the idea and designed the analytical framework for this work. PJH implemented the design, collected data, and analysed results. Both JYC and AML provided timely guidance and critical feedback throughout the research. The manuscript was drafted by PJH and carefully revised by JYC and AML.

\section{Competing interests}

Jake Chen is the co-founder of Medeolinx, LLC, a company dedicated to providing bioinformatics solutions to pharmaceutical companies engaging in predictive and personalized medicine. Jake Chen declares no conflict of interest for this work.

Published: 17 December 2012

\section{References}

1. Wheeler E, Barroso I: Genome-wide association studies and type 2 diabetes. Brief Funct Genomics 2011, 10(2):52-60.

2. Cruz M, Valladares-Salgado A, Garcia-Mena J, Ross K, Edwards M, AngelesMartinez J, Ortega-Camarillo C, de la Pena JE, Burguete-Garcia Al, WacherRodarte N, et al: Candidate gene association study conditioning on individual ancestry in patients with type 2 diabetes and metabolic syndrome from Mexico City. Diabetes Metab Res Rev 2010, 26(4):261-270.

3. Lin $Y$, Li $P$, Cai $L$, Zhang B, Tang $X$, Zhang $X$, Li $Y$, Xian $Y$, Yang $Y$, Wang $L$, et al: Association study of genetic variants in eight genes/loci with type 2 diabetes in a Han Chinese population. BMC Med Genet 2010, 11:97.

4. Sladek R, Rocheleau G, Rung J, Dina C, Shen L, Serre D, Boutin P, Vincent D, Belisle A, Hadjadj S, et al: A genome-wide association study identifies novel risk loci for type 2 diabetes. Nature 2007, 445(7130):881-885.
5. Gaulton KJ, Willer CJ, Li Y, Scott LJ, Conneely KN, Jackson AU, Duren WL, Chines PS, Narisu N, Bonnycastle LL, et al: Comprehensive association study of type 2 diabetes and related quantitative traits with 222 candidate genes. Diabetes 2008, 57(11):3136-3144.

6. Hu C, Zhang R, Wang C, Wang J, Ma X, Lu J, Qin W, Hou X, Bao Y, Xiang K, et al: PPARG, KCNJ11, CDKAL1, CDKN2A-CDKN2B, IDE-KIF11-HHEX, IGF2BP2 and SLC30A8 are associated with type 2 diabetes in a Chinese population. PLoS One 2009, 4(10):e7643.

7. Scott L, Mohlke KL, Bonnycastle LL, Willer CJ, Li Y, Duren WL, Erdos MR, Stringham HM, Chines PS, Jackson AU, et al: A genome-wide association study of type 2 diabetes in Finns detects multiple susceptibility variants. Science 2007, 316(5829):1341-1345.

8. So HC, Gui AH, Cherny SS, Sham PC: Evaluating the heritability explained by known susceptibility variants: a survey of ten complex diseases. Genet Epidemiol 2011, 35(5):310-317.

9. Cauchi S, Meyre D, Durand E, Proenca C, Marre M, Hadjadj S, Choquet H, De Graeve F, Gaget $S$, Allegaert F, et al: Post genome-wide association studies of novel genes associated with type 2 diabetes show gene-gene interaction and high predictive value. PLoS One 2008, 3(5):e2031.

10. Sharma A, Chavali S, Tabassum R, Tandon N, Bharadwaj D: Gene prioritization in Type 2 Diabetes using domain interactions and network analysis. BMC Genomics 2010, 11:84

11. Jesmin J, Rashid MS, Jamil H, Hontecillas R, Bassaganya-Riera J: Gene regulatory network reveals oxidative stress as the underlying molecular mechanism of type 2 diabetes and hypertension. BMC Med Genomics 2010, 3(1):45.

12. Lim JE, Hong KW, Jin HS, Kim YS, Park HK, Oh B: Type 2 diabetes genetic association database manually curated for the study design and odds ratio. BMC Med Inform Decis Mak 2010, 10:76.

13. Sherry ST, Ward MH, Kholodov M, Baker J, Phan L, Smigielski EM, Sirotkin K: dbSNP: the NCBI database of genetic variation. Nucleic acids research 2001, 29(1):308-311.

14. Chen JY, Mamidipalli S, Huan T: HAPPI: an online database of comprehensive human annotated and predicted protein interactions. BMC genomics 2009, 10(Suppl 1):S16.

15. Loveland J: VEGA, the genome browser with a difference. Brief Bioinform 2005, 6(2):189-193.

16. von Mering $C$, Jensen $L$, Kuhn M, Chaffron S, Doerks T, Kruger B, Snel B, Bork P: STRING 7-recent developments in the integration and prediction of protein interactions. Nucleic acids research 2007, 35(Database):D358-362

17. Brown KR, Jurisica I: Online predicted human interaction database. Bioinformatics 2005, 21(9):2076-2082.

18. Alfarano C, Andrade CE, Anthony K, Bahroos N, Bajec M, Bantoft K, Betel D,

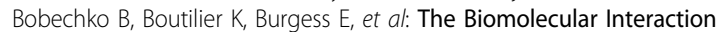
Network Database and related tools 2005 update. Nucleic acids research 2005, 33(Database):D418-424.

19. Mishra GR, Suresh M, Kumaran K, Kannabiran N, Suresh S, Bala P, Shivakumar K, Anuradha N, Reddy R, Raghavan TM, et al: Human protein reference database-2006 update. Nucleic acids research 2006, 34(Database):D411-414. 
20. Chatr-aryamontri A, Ceol A, Palazzi LM, Nardelli G, Schneider MV, Castagnoli L, Cesareni G: MINT: the Molecular INTeraction database. Nucleic acids research 2007, 35(Database):D572-574.

21. Li J, Zhu X, Chen JY: Building disease-specific drug-protein connectivity maps from molecular interaction networks and PubMed abstracts. PLoS Comput Biol 2009, 5(7):e1000450.

22. Huang da W, Sherman BT, Lempicki RA: Bioinformatics enrichment tools: paths toward the comprehensive functional analysis of large gene lists. Nucleic acids research 2009, 37(1):1-13.

23. Huang da W, Sherman BT, Lempicki RA: Systematic and integrative analysis of large gene lists using DAVID bioinformatics resources. Nat Protoc 2009, 4(1):44-57.

24. Kanehisa M, Goto S, Sato Y, Furumichi M, Tanabe M: KEGG for integration and interpretation of large-scale molecular data sets. Nucleic acids research 2012, 40(Database):D109-114

25. Rho K, Kim B, Jang Y, Lee S, Bae T, Seo J, Seo C, Lee J, Kang H, Yu U, et al: GARNET-gene set analysis with exploration of annotation relations. BMC Bioinformatics 2011, 12(Suppl 1):S25.

26. Ji L, Malecki M, Warram JH, Yang Y, Rich SS, Krolewski AS: New susceptibility locus for NIDDM is localized to human chromosome $20 \mathrm{q}$. Diabetes 1997, 46(5):876-881.

27. Zouali H, Hani EH, Philippi A, Vionnet N, Beckmann JS, Demenais F, Froguel P: A susceptibility locus for early-onset non-insulin dependent (type 2) diabetes mellitus maps to chromosome $20 \mathrm{q}$, proximal to the phosphoenolpyruvate carboxykinase gene. Hum Mol Genet 1997. 6(9):1401-1408

28. Sandovici I, Smith NH, Nitert MD, Ackers-Johnson M, Uribe-Lewis $S$, Ito $Y$ Jones RH, Marquez VE, Cairns W, Tadayyon M, et al: Maternal diet and aging alter the epigenetic control of a promoter-enhancer interaction at the Hnf4a gene in rat pancreatic islets. Proc Natl Acad Sci USA 2011, 108(13):5449-5454.

29. Barroso I, Luan J, Middelberg RP, Harding AH, Franks PW, Jakes RW, Clayton D, Schafer AJ, O'Rahilly S, Wareham NJ: Candidate gene association study in type 2 diabetes indicates a role for genes involved in beta-cell function as well as insulin action. PLoS Biol 2003, 1(1):E20.

30. Malodobra M, Pilecka A, Gworys B, Adamiec R: Single nucleotide polymorphisms within functional regions of genes implicated in insulin action and association with the insulin resistant phenotype. Mol Cell Biochem 2011, 349(1-2):187-193.

31. Rasche A, Al-Hasani H, Herwig R: Meta-analysis approach identifies candidate genes and associated molecular networks for type-2 diabetes mellitus. BMC Genomics 2008, 9:310

32. Huang $Q$, Wang TH, Lu WS, Mu PW, Yang YF, Liang WW, Li CX, Lin GP. Estrogen receptor alpha gene polymorphism associated with type 2 diabetes mellitus and the serum lipid concentration in Chinese women in Guangzhou. Chin Med J (Engl) 2006, 119(21):1794-1801.

33. Dahlman I, Vaxillaire M, Nilsson M, Lecoeur C, Gu HF, Cavalcanti-Proenca $C$ Efendic S, Ostenson CG, Brismar K, Charpentier G, et al: Estrogen receptor alpha gene variants associate with type 2 diabetes and fasting plasma glucose. Pharmacogenet Genomics 2008, 18(11):967-975.

34. Di Paola R, Caporarello N, Marucci A, Dimatteo C, ladicicco C, Del Guerra S, Prudente S, Sudano D, Miele C, Parrino C, et al: ENPP1 affects insulin action and secretion: evidences from in vitro studies. PLOS One 2011, 6(5):e19462.

35. Wang J, Kuusisto J, Vanttinen M, Kuulasmaa T, Lindstrom J, Tuomilehto J, Uusitupa M, Laakso M: Variants of transcription factor 7-like 2 (TCF7L2) gene predict conversion to type 2 diabetes in the Finnish Diabetes Prevention Study and are associated with impaired glucose regulation and impaired insulin secretion. Diabetologia 2007, 50(6):1192-1200.

36. Horikoshi M, Hara K, Ito C, Nagai R, Froguel P, Kadowaki T: A genetic variation of the transcription factor 7 -like 2 gene is associated with risk of type 2 diabetes in the Japanese population. Diabetologia 2007, 50(4):747-751.

37. Ren Q, Han XY, Wang F, Zhang XY, Han LC, Luo YY, Zhou XH, Ji LN: Exon sequencing and association analysis of polymorphisms in TCF7L2 with type 2 diabetes in a Chinese population. Diabetologia 2008, 51(7):1146-1152

38. Guo T, Hanson RL, Traurig M, Muller YL, Ma L, Mack J, Kobes S, Knowler WC, Bogardus C, Baier LJ: TCF7L2 is not a major susceptibility gene for type 2 diabetes in Pima Indians: analysis of 3,501 individuals. Diabetes 2007. 56(12):3082-3088.
doi:10.1186/1752-0509-6-S3-S16

Cite this article as: Hale et al:: Genome-wide meta-analysis of genetic susceptible genes for Type 2 Diabetes. BMC Systems Biology 2012 6(Suppl 3):S16

\section{Submit your next manuscript to BioMed Central and take full advantage of:}

- Convenient online submission

- Thorough peer review

- No space constraints or color figure charges

- Immediate publication on acceptance

- Inclusion in PubMed, CAS, Scopus and Google Scholar

- Research which is freely available for redistribution
C) Biomed Central 